\title{
El mes de recolección de la macha (Mesodesma donacium) determinado por sus líneas de crecimiento: aplicaciones arqueológicas
}

\section{Matthieu Carré}

\section{(2) OpenEdition Journals \\ Edición electrónica \\ URL: http://journals.openedition.org/bifea/3983 \\ DOI: 10.4000/bifea.3983 \\ ISSN: 2076-5827 \\ Editor \\ Institut Français d'Études Andines}

\section{Edición impresa}

Fecha de publicación: 1 agosto 2007

Paginación: 299-304

ISSN: 0303-7495

\section{Referencia electrónica}

Matthieu Carré, «El mes de recolección de la macha (Mesodesma donacium) determinado por sus líneas de crecimiento: aplicaciones arqueológicas », Bulletin de l'Institut français d'études andines [En línea], 36 (2) | 2007, Publicado el 01 febrero 2008, consultado el 01 diciembre 2020. URL : http:// journals.openedition.org/bifea/3983; DOI : https://doi.org/10.4000/bifea.3983

\section{(c) $(7)$}

Les contenus du Bulletin de l'Institut français d'études andines sont mis à disposition selon les termes de la licence Creative Commons Attribution - Pas d'Utilisation Commerciale - Pas de Modification 4.0 International. 


\title{
El mes de recolección de la macha (Mesodesma donacium) determinado por sus líneas de crecimiento: aplicaciones arqueológicas
}

\author{
Matthieu Carré*
}

\section{INTRODUCCIÓN}

La macha (Mesodesma donacium) tiene una importancia económica desde que llegaron los primeros pescadores recolectores a la costa peruana hace 11000 años. Ciertos conchales constituidos casi exclusivamente de las conchas de este bivalvo forman montículos de hasta diez metros de alto y atestiguan de una larga e intensa explotación pasada (Engel, 1957; Sandweiss et al., 1989; 1998; Lavallée et al., 1999). Los arqueólogos disponen en general de muy pocos índices para determinar si los sitios arqueológicos estaban ocupados de manera permanente o estacional, especialmente para el periodo precerámico. Aquí se presenta un método utilizando las líneas de crecimiento de la concha de M. donacium, para determinar el mes de recolección de cada concha.

\section{CICLOS DE CRECIMIENTO}

M. donacium es un bivalvo filtrador que vive en las playas arenosas de Perú y Chile desde la banda intermareal hasta 15 metros de profundidad. Hasta 1982, su repartición geográfica iba de la isla Chiloe $\left(\sim 42^{\circ} \mathrm{S}\right)$ hasta la Bahia de Sechura $\left(\sim 6^{\circ} \mathrm{S}\right)$ (Tarifeño, 1980). Por su excepcional intensidad, los eventos El Niño de 1982-1983 y de 1997-1998 provocaron una mortalidad muy

* University of Washington, School of Oceanography, Box 355351, Seattle, WA 98195, USA.

E-mail: mcarre@u.washington.edu 
alta de esta especie que, hasta hoy, desapareció al norte de $15^{\circ} \mathrm{S}$ (Barriga \& Quiroz, 2002). Sin embargo, esa no es la consecuencia usual de un El Niño, pues la especie se mantuvo hasta $6^{\circ} \mathrm{S}$ a pesar de que el fenómeno siempre fue activo en los últimos siglos (Cobb et al., 2003). Hoy en día pescadores bucean para recolectar las machas, pero tradicionalmente se recolecta a pie a poca profundidad durante la marea baja. Estas conchas de poca profundidad están muy influenciadas por los ciclos de marea. Cuando el mar está a su nivel más bajo, la concha se cierra y forma una capa con mayor concentración de materia orgánica (principalmente proteinas) que aparece en sección como una línea más oscura. Según la amplitud de marea, una o dos líneas se forman así por día. Los coeficientes de marea son mayores durante las fases de luna llena o de luna nueva (mareas vivas), lo que se traduce en la concha por grupos de líneas más gruesas y menos separadas. Estos grupos se forman con una periodicidad de un medio mes lunar es decir entre 12 y 16 días, con un promedio de 14 días. Cuando la tasa de crecimiento de la concha está baja, las líneas diarias no se pueden distinguir, y los grupos bisemanales aparecen como líneas más o menos marcadas (Fig. 1). M. donacium es una especie típica de las aguas frias de la corriente de Humboldt. En el Perú, su tasa de crecimiento disminuye durante la época más cálida, de enero a marzo, lo que produce en las conchas un banda de verano oscura y ancha (más detalles en Carré et al., 2005a).

\section{DETERMINAR EL MES DE RECOLECCIÓN}

\section{1. Preparación de la concha}

Se escogen conchas de al menos $50 \mathrm{~mm}$ de largo. La mayoría de las conchas están quebradas en los yacimientos arqueológicos. Sin embargo, se puede usar los fragmentos que permiten hacer un corte recto desde la charnela hasta el borde. Se encajan las conchas en resina polyester y se corta una sección radial de $\sim 1 \mathrm{~mm}$ de espesor con una sierra de corte lento. Se pega con resina epoxy la sección en una lámina de vidrio y se pule la superficie. Las líneas de crecimiento de esta especie se pueden observar sin más preparación con un microscopio binocular y luz directa.

\section{2. Método}

Las líneas de crecimiento representan una cronología (sclerocronología) desde la parte juvenil (el umbo) hasta la muerte del animal (el borde). Primero se debe identificar la última banda de verano en la sección de concha, que será la referencia temporal. Después de estudiar el registro de temperatura de los 30 últimos años en Ilo, en el sur del Perú, hemos decidido atribuir al límite final de la banda de verano la fecha 15 de marzo, que corresponde en promedio al inicio de bajada de la temperatura del mar. En otras regiones, se deberá estudiar registros locales de temperatura del mar para comprobar que esta fecha es adecuada (un mes después de la fecha promedio del máximo de temperatura). A partir de esta referencia, se identifica y se cuenta el número $\mathrm{N}$ de ciclos bisemanales hasta el borde de la concha (Fig. 1A). La fecha de recolección se calcula sabiendo que ocurrió $14 \times \mathrm{N}$ días después del 15 de marzo.

\section{INCERTIDUMBRES}

\section{1. Marca anual}

Existe mucha variabilidad en las líneas de crecimiento de las conchas, lo que complica la identificación de las bandas de verano y de los ciclos bisemanales. Durante muchos veranos en la 


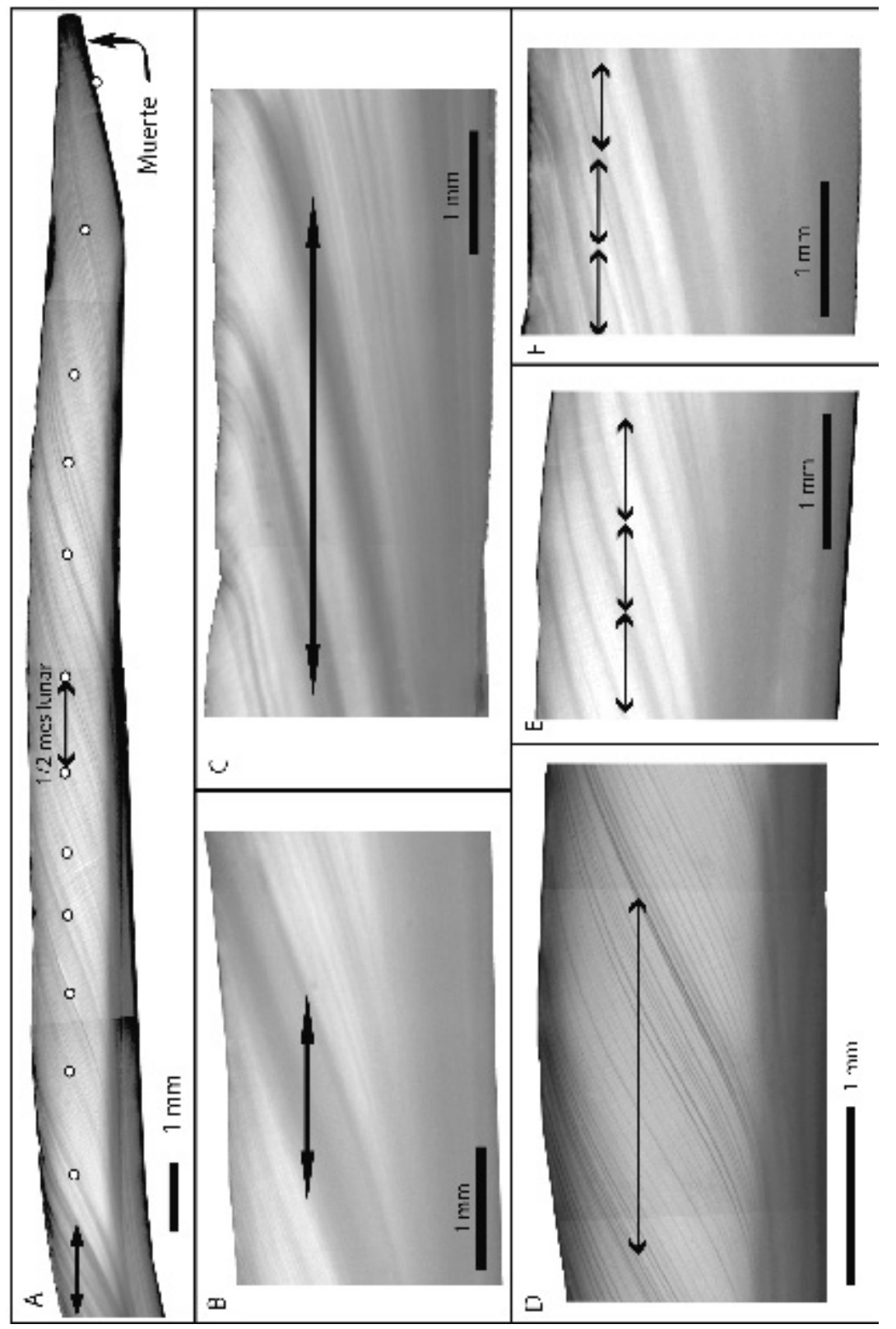

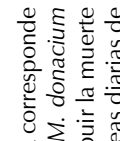

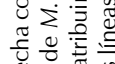

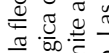

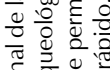

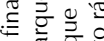

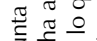

등

O)

ฮิ

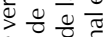

ㄴํㅇ ㅇำ

穷芯造造

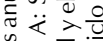

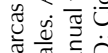

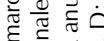

疍

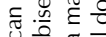

을 은

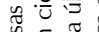

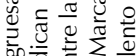

ช.

ट्र

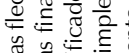

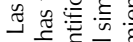

过

ब

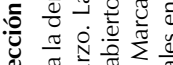

造 $\frac{\pi}{\pi} \frac{\pi}{2}$

ฮ 두잉

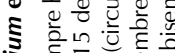

:

ธक ज

ป छ

¿ 0 己

원 능 는

:

范

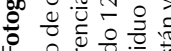

난은 원 $\frac{0}{2}$

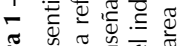

ฐ 
costa peruana, la temperatura del mar no pasa por un máximo sino por dos. Así una sola estación de verano puede estar representada por una o dos bandas oscuras (Fig. 1B, C). Una banda puede también ser producida por el desove que occurre por noviembre (Brown \& Guerra, 1979). La fecha de referencia se atribuye al límite final de la última marca. Eventos climáticos como tormentas pueden también provocar un paro de crecimiento y una banda oscura, pero generalmente más fina, más oscura, sin transición. La fecha de referencia (aquí escogida como 15 de marzo) tiene una incertidumbre relacionada a la variabilidad climática. Calculando la desviación estándar del máximo anual de temperatura, se evaluó esta incertidumbre a \pm 1 mes $(1 \sigma)$.

\section{2. Identificación de los ciclos de marea}

El número $\mathrm{N}$ de ciclos bisemanales tiene una incertidumbre relacionada a la dificultad de identificar estos ciclos. La variabilidad de apariencia de los grupos de líneas que corresponden a las mareas vivas tiene varias causas: su tamaño depende de la tasa de crecimiento; los ciclos de marea son variables; el estado del mar (sereno o agitado) modula la influencia de las mareas; eventos de efecto corto interfieren (tormenta, depredado). Observar con diferentes ampliaciones ayuda en general mucho para la determinación. Sin embargo, por la duda que siempre existe, el número de ciclos se debe considerar en un intervalo [N-2, N+2]. La incertidumbre asociada

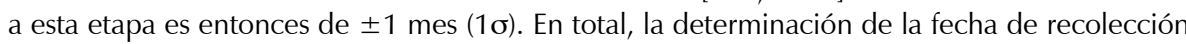
tiene una incertidumbre de \pm 2 meses.

\section{3. Casos de Indeterminación}

No siempre es posible determinar una fecha de recolección por 3 razones principales:

- si el individuo es muy joven (menos de un año), no habrá formado una marca anual de verano;

- si el individuo es muy viejo (a partir de 3 ó 4 años), la tasa de crecimiento cerca del borde de la concha es demasiado baja para distinguir y contar las estructuras de crecimiento;

- si el estado de preservación de la concha arqueológica es malo, la materia orgánica está destruida y las líneas de crecimiento ya no aparecen.

Estos casos de indeterminación no son susceptibles de inducir un bies en la distribución de frecuencias.

\section{VALIDEZ TEMPORAL Y ESPACIAL}

Para validar el método en condiciones climáticas diferentes, comprobar su exactitud y su incertidumbre, se comparó, en 12 conchas fósiles, las fechas de recolección determinadas por las líneas de crecimiento con las fechas determinadas por curvas isotópicas $\left(\square^{18} \mathrm{O}\right)$ que representan las variaciones de temperatura del mar (Carré et al., 2005a). La fecha se estima por el fin de la curva estacional de temperatura. El método isotópico es muy confiable pero su costo impide analizar muchas conchas. La diferencia de fecha entre los dos métodos tiene un promedio de 0,6 mes y una desviación estándar de 1,8 meses (Carré, 2005), lo que confirma que la determinación con las líneas de crecimiento es exacta, con una precisión cerca de \pm 2 meses.

Además, los resultados isotópicos enseñaron que la temperatura del mar en el sur del Perú estaba $3^{\circ} \mathrm{C}$ más baja que hoy en la época estudiada (9 000 a 7000 años antes del presente) (Carré et al., 2005b). Este estudio comparativo nos permitió demostrar que el método es también válido en condiciones climáticas diferentes. Finalmente, este resultado sugiere que el método que hemos presentado se puede aplicar en conchas de $M$. donacium que han vivido en zonas geográficas 
o en épocas donde la temperatura promedio está entre 13 y $16^{\circ} \mathrm{C}$. Hoy, eso representa toda la costa peruana al sur de $7^{\circ} \mathrm{S}$, hasta Chile central. Para un estudio en la parte más fria de la zona de repartición, será necesario estudiar primero las condiciones de formación de las líneas anuales que son probablemente muy diferentes, pues la temperatura limitante es posiblemente la de invierno austral y el desove podría también ocurrir a otro momento del año. Se recomienda un corto estudio isotópico de las conchas arqueológicas que dé indicaciones de las condiciones promedio de temperatura, a partir de la ecuación de paleotemperatura publicada por Carré et al. (2005a).

\section{INFLUENCIA DE EL NIÑO}

El fenómeno climático con mayor impacto ambiental y económico en la costa peruana es $\mathrm{El}$ Niño. Su influencia en la evolución de las civilizaciones costeras es un tema muy importante en el cual los moluscos pueden ser archivos muy valiosos (Sandweiss et al., 2001; Carré et al., 2005a; b). Sin embargo, el efecto de un evento en el crecimiento de una concha de $M$. donacium es muy variable (desde una marca de verano «normal» hasta la muerte) y depende: i) de la zona geográfica; ii) de la intensidad del evento; iii) de la edad del individuo, lo que no permite hacer un diagnóstico confiable por anomalías de crecimiento.

Sin embargo, la actividad de El Niño se puede estudiar reconstruyendo las temperaturas mensuales del mar registradas por los isotopos estables del oxígeno $\left(\square^{18} \mathrm{O}\right)$ en las conchas de $M$. donacium (Carré et al., 2005a; b; Carré, 2005).

\section{CONCLUSIÓN}

Se presentó un nuevo método para determinar el mes de muerte de las conchas de M. donacium por el análisis de las estructuras periódicas de sus líneas de crecimiento. La incertidumbre de este método es de \pm 2 meses, lo que representa un aumento de precisión importante comparado a la mayoría de los estudios similares utilizando conchas de moluscos que solo determinan la estación de recolección (invierno/verano) (Quitmyer et al., 1997). Reproduciendo este análisis en una gran cantidad de conchas de un nivel arqueológico, resulta una curva mensual de frecuencia de pesca para el periodo estudiado. Este método se puede aplicar a todos los sitios arqueológicos de la costa peruana y del norte de Chile conteniendo conchas de $M$. donacium, si la temperatura promedio en la época estudiada está entre 13 y $16^{\circ} \mathrm{C}$. Se indicaron los principios metodológicos para adaptar el método a los sitios de la parte sur de Chile. Los arqueólogos disponen de una nueva herramienta para determinar la estacionalidad de actividad de recolección de esta especie en el pasado. Esta información es de mayor importancia para estudiar las estrategias de explotación de los recursos, y los patrones de movilidad de los grupos de pescadores.

\section{Agradecimientos}

Agradecemos al Dr. Daniel Sandweiss y al Dr. Víctor Vásquez Sánchez por sus comentarios pertinentes y a Nancy Mitma García por su colaboración en la redacción. Agradecemos a Danièle Lavallée, Michel Fontugne y Luc Ortlieb por su ayuda para conseguir muestras de machas modernas, y el proyecto Pérou-Sud (dir. Danièle Lavallée y Michèle Julien) por las conchas arqueológicas de la Quebrada de los Burros. Agradecemos a Marco Quiroz Ruiz y a Fredy Cardenas del IMARPE de Ilo. 


\section{Referencias citadas}

BARRIGA, E. \& QUIROZ, M., 2002 - Impacts of El Niño and La Niña events on the populations of clam (Mesodesma donacium, Lamarck 1818) along the southern Peruvian coast, Investigaciones Marinas, 30 (1): 134-135.

BROWN, D. \& GUERRA, R., 1979 - Etapas en el desarrollo de la «macha», Mesodesma donacium (Lamarck, 1818) en la bahías de Guanaqueros y Coquimbo, 29 p.; Coquimbo: Centro de Investigaciones Submarinas, Universidad del Norte.

CARRÉ, M., 2005 - Étude géochimique et sclérochronologique de coquilles de bivalves marins : paléocéanographie de la côte sud du Pérou à l'Holocène inférieur et implications archéologiques; Université Montpellier 2, 348 p. Tesis de doctorado no publicada

CARRÉ, M., BENTALEB, I., BLAMART, D., OGLE, N., CÁRDENAS, F., ZEVALLOS, S., KALIN, R.M., ORTLIEB, L. \& FONTUGNE, M., 2005a - Stable isotopes and sclerochronology of the bivalve Mesodesma donacium: potential application to peruvian paleoceanographic reconstructions. Palaeogeography, Palaeoclimatology, Palaeoecology, 228: 4-25.

CARRÉ, M., BENTALEB, I., FONTUGNE, M. \& LAVALLÉE, D., 2005b - Strong El Niño events during the early Holocene: stable isotope evidence from Peruvian sea-shells. The Holocene, 15 (1): 42-47.

COBB, K.M., CHARLES, C.D., CHENG, H. \& EDWARDS, R.L., 2003 - El Niño/Southern Oscillation and tropical Pacific climate during the last millenium. Nature, 424: 271 276.

LAVALlÉE, D., JULIEN, M., BÉAREZ, P., USSELMAN, P., FONTUGNE, M. \& BOLAÑOS, A., 1999 - Pescadores-recolectores arcaicos del extremo-sur Peruano. Excavaciones en la Quebrada de los Burros (departamento de Tacna). Primeros resultados 1995-1997. Bulletin de l'Institut Français d'Études Andines, 28 (1): 13-52.

ENGEL, F., 1957 - Early sites on the Peruvian coast. Southwestern Journal of Anthropology, 13 (1): 54-68.

QUITMYER, I.R., JONES, D.S. \& ARNOLD, W.S., 1997 - The sclerochronology of hard clams, Mercenaria spp., from the south-eastern U.S.A.: a method of elucidating the zooarchaeological records of seasonal resource procurement and seasonality in prehistoric shell middens. Journal of Archaeological Science, 24: 825-840.

SANDWEISS, D.H., RICHARDSON III, J.B., REITZ, E.J., HSU, J.T. \& FELDMAN, R.A., 1989 - Early maritime adaptations in the Andes: preliminary studies at the Ring Site, Peru. In: Ecology, Settlement and History in the Osmore Drainage, Peru (D. Rice, C. Stanish \& P.R. Scarr): 35-84; Oxford: BAR International Series 545 (i).

SANDWEISS, D.H., MCINNIS, H., BURGER, R.L., CANO, A., OJEDA, B., PAREDES, R., SANDWEISS, M.C. \& GLASCOCK, M.D., 1998 - Quebrada Jaguay: South American maritime adaptations. Science, 281: 1830-1832.

SANDWEISS, D.H., MAASCH, K.A., BURGER, R.L., RICHARDSON III, J.B., ROLLINS, H.B., CLEMENT, A., 2001 - Variation in Holocene El Niño frequencies: Climate records and cultural consequences in ancient Peru. Geology, 29 (7): 603-606.

TARIFEÑO, E., 1980 - Studies on the biology of surf clam Mesodesma donacium (Lamarck, 1818) (Bivalvia: Mesodesmatidae) from Chilean sandy beaches, 229 p.; Los Angeles: University of California. 